\title{
COMMENTARY ON "NUMBERS OF SOLUTIONS OF EQUATIONS IN FINITE FIELDS" BY ANDRÉ WEIL
}

\begin{abstract}
MARK GORESKY
ABSTRACT. Immediately following the commentary below, this previously published article is reprinted in its entirety: André Weil, "Numbers and solutions of equations in finite fields", Bull. Amer. Math. Soc., 55 (1949), no. 5, 497508 .
\end{abstract}

The paper "Numbers of solutions of equations in finite fields" of André Weil W1] ranks among the most influential articles in the mathematics of the last hundred years. On the last page of this paper Weil proposes a collection of precise conjectures which initiated a vast development in mathematics.

It would require a much longer article than these few paragraphs to describe the evolution of the "Weil conjectures". The Wikipedia article [Wik] is a good starting point, perhaps followed by J. Milne's comprehensive study [M]. See also $\mathrm{Di}, \mathrm{H}, \mathrm{O}, \mathrm{K}$.

Weil's article begins with a laborious computation of the number of solutions over a finite field to a certain equation $\sum a_{r} x^{r}=0$. Initially, Weil offers a somewhat strained motivation for doing this calculation, and (other than in the title of the article) he gives no indication that he is interested in situations more general than this particular equation. However, at the end of the article the real point of the calculation is revealed: it is a nontrivial example which he uses to motivate the conjectures. Weil then states that "this, and many other examples that we cannot discuss here" support the conjectures. He had clearly been thinking about this circle of ideas for some time.

Weil's conjectures were revolutionary because they proposed a precise and deep relation between two completely different sorts of objects: the topology of a complex algebraic variety versus the number of points $(\bmod p)$ in that variety. For most mathematicians this probably looked like a message from outer space, and Carlitz in his Mathematical Reviews article does little more than quote, without comment, Weil's sentence that introduces the conjectures.

In his 1954 ICM lecture [W2, perhaps as a result of daydreaming about these questions for the intervening five years, Weil points out that his conjectures could be conveniently rephrased in terms of the Lefschetz fixed point formula for the self-map that is defined by the Frobenius morphism, thus cementing the relation between topology and number theory that he had proposed in the Bulletin article. He does not discuss the obvious problem that a new cohomology theory (valid for

Received by the editors March 16, 2018.

2010 Mathematics Subject Classification. Primary 11G25, 11M38, 14F20. 
algebraic varieties defined over finite fields) would be needed in order to make sense of such a Lefschetz formula.

Soon after Weil's talk at the ICM, J. P. Serre found an example proving that such a cohomology theory, with rational coefficients, could not exist. Some people suspect that Weil may have resisted the idea of a "Weil cohomology theory" for years. His skepticism may have been reinforced when, in 1960, B. Dwork Dw proved the first of the conjectures (rationality of the zeta function) using (noncohomological) $p$-adic methods. Nevertheless the whole picture was so compelling that, at some point between 1954 and 1958, mathematicians began to actively seek a cohomology theory in which Weil's conjectures could be formulated and proven. In 1958 Serre published [S] a reasonable but ultimately unsuccessful attempt. In 1958 Grothendieck G1 made a first outline of a general theory, and by 1972 [A] M. Artin and A. Grothendieck had succeeded in constructing the required étale cohomology theory. Together with J. L. Verdier they used it to prove the first three of Weil's conjectures. (The theory was later simplified by Deligne [D3.)

Applications of these results were immediate. For example, even before the last of the Weil conjectures had been established, Deligne showed D2 that, if true, it could then be used to prove the Ramanujan conjecture (and its generalization, the Ramnujan-Petersson conjecture) from the theory of modular forms.

The remarkable collection of mathematical ideas involved in Deligne's proof [D1] of the fourth conjecture is described in $[\mathrm{K}, \mathrm{O}]$. In the current literature, applications of the "Weil conjectures" (that is, the resulting theorems of Artin, Deligne, Grothendieck, and Verdier) have become so common that many authors simply cite "Weil conjectures" without reference, whenever these results are used.

\section{REFERENCES}

[A] Théorie des topos et cohomologie étale des schémas. Tome 1: Théorie des topos (French), Lecture Notes in Mathematics, Vol. 269, Springer-Verlag, Berlin-New York, 1972. Séminaire de Géométrie Algébrique du Bois-Marie 1963-1964 (SGA 4); Dirigé par M. Artin, A. Grothendieck, et J. L. Verdier. Avec la collaboration de N. Bourbaki, P. Deligne, et B. Saint-Donat. MR 0354652

[D1] P. Deligne, La conjecture de Weil. I (French), Inst. Hautes Études Sci. Publ. Math. 43 (1974), 273-307. MR0340258

[D2] P. Deligne, Formes modulaires et représentations l-adiques (French), Séminaire Bourbaki. Vol. 1968/69: Exposés 347-363, Lecture Notes in Math., vol. 175, Springer, Berlin, 1971, pp. Exp. No. 355, 139-172. MR3077124

[D3] P. Deligne, Cohomologie étale (French), Lecture Notes in Mathematics, vol. 569, SpringerVerlag, Berlin, 1977. Séminaire de géométrie algébrique du Bois-Marie SGA $4 \frac{1}{2}$. MR 463174

[Di] J. Dieudonné, On the history of the Weil conjectures, in Etale Cohomology and the Weil Conjecture edited by E. Freitag and R. Kiehl, Ergeb. 13, Springer Verlag, N.Y., 1987.

[Dw] B. Dwork, On the rationality of the zeta function of an algebraic variety, Amer. J. Math. 82 (1960), 631-648, DOI 10.2307/2372974. MR0140494

[G1] A. Grothendieck, The cohomology theory of abstract algebraic varieties, Proc. Internat. Congress Math. (Edinburgh, 1958), Cambridge University Press, New York, 1960, pp. 103118. MR0130879

[H] R. Hartshorne, Algebraic geometry, Springer-Verlag, New York-Heidelberg, 1977. Graduate Texts in Mathematics, No. 52. MR0463157

[K] N. M. Katz, An overview of Deligne's proof of the Riemann hypothesis for varieties over finite fields, Mathematical developments arising from Hilbert problems (Proc. Sympos. Pure Math., Vol. XXVIII, Northern Illinois Univ., De Kalb, Ill., 1974), Amer. Math. Soc., Providence, R.I., 1976, pp. 275-305. See The Weil conjectutes, Appendix C. MR0424822 
[M] J. S. Milne, The Riemann hypothesis over finite fields from Weil to the present day, The legacy of Bernhard Riemann after one hundred and fifty years. Vol. II, Adv. Lect. Math. (ALM), vol. 35, Int. Press, Somerville, MA, 2016, pp. 487-565. MR3525903

[O] F. Oort, The Weil conjectures, Nieuw Arch. Wiskd. (5) 15 (2014), no. 3, 211-219. MR 3243075

[S] J.-P. Serre, Sur la topologie des variétés algébriques en caractéristique p (French), Symposium internacional de topología algebraica (International symposium on algebraic topology), Universidad Nacional Autónoma de México and UNESCO, Mexico City, 1958, pp. 24-53. MR0098097

[W1] A. Weil, Numbers of solutions of equations in finite fields, Bull. Amer. Math. Soc. 55 (1949), 497-508, DOI 10.1090/S0002-9904-1949-09219-4. MR0029393

[W2] A. Weil, Abstract versus classical algebraic geometry, Proceedings of the International Congress of Mathematicians, 1954, Amsterdam, vol. III, Erven P. Noordhoff N.V., Groningen; North-Holland Publishing Co., Amsterdam, 1956, pp. 550-558. MR0092196

[Wik] The Weil Conjectures, Wikipedia. Retrieved March 10, 2018.

School of Mathematics, Institute for Advanced Study, Princeton, New Jersey

Email address: goresky@ias.edu 